\title{
COEXISTENCE AND NONCOEXISTENCE OF MARKOVIAN VIRUSES AND THEIR HOSTS
}

\author{
JAKOB E. BJÖRNBERG * AND \\ ERIK I. BROMAN, *** Uppsala University
}

\begin{abstract}
Examining possibilities for the coexistence of two competing populations is a classic problem which dates back to the earliest 'predator-prey' models. In this paper we study this problem in the context of a model introduced in Björnberg et al. (2012) for the spread of a virus infection in a population of healthy cells. The infected cells may be seen as a population of 'predators' and the healthy cells as a population of 'prey'. We show that, depending on the parameters defining the model, there may or may not be coexistence of the two populations, and we give precise criteria for this.
\end{abstract}

Keywords: Coexistence; branching process; interacting branching process

2010 Mathematics Subject Classification: Primary 60J80

Secondary $60 \mathrm{~J} 85$

\section{Introduction}

We start by giving an informal description of the model studied in this paper. The model consists of a two-dimensional Markov process $(X(t), Y(t))_{t \geq 0}$, where $X(t)$ is the number of 'healthy cells' at time $t$, and $Y(t)$ is the number of 'infected cells' (i.e. cells containing a virus). Both the $(X(t))_{t \geq 0}$ and $(Y(t))_{t \geq 0}$ components behave in many ways like branching processes, although there are dependencies between them. A healthy cell is replaced by a random number of new healthy cells at rate 1 . This random number is independent of other events and drawn from a distribution $\left(p_{k}\right)_{k \geq 0}$; thus, the rate at which a healthy cell is replaced by $k$ healthy cells is $p_{k}$. Infected cells are also replaced by $k$ new (infected) cells at rate $p_{k}$ if $k \geq 1$, while they are replaced by 0 new cells (die) at the higher rate $p_{0}+\lambda$. Here $\lambda \geq 0$ is a parameter that reflects the negative impact of the virus on the host's life span. When an infected cell dies (i.e. is replaced by 0 new cells), it converts a random number of healthy cells into infected cells. The biological motivation is that, when infected cells die, they burst and release 'free virions' which enter a random number of healthy cells, thus infecting them. This process is called a lysis in biology (see also Section 2). The number of conversions is independent of all other events, and is drawn from a distribution $\left(\gamma_{k}\right)_{k \geq 0}$. Hence, the processes $(X(t))_{t \geq 0}$ and $(Y(t))_{t \geq 0}$ interact in the sense that $(Y(t))_{t \geq 0}$ 'feeds' upon $(X(t))_{t \geq 0}$. The model is defined in detail in Section 2. We refer the interested reader to [3] for a biological motivation of the model. We will sometimes simply write $X$ or $Y$ as a shorthand for $(X(t))_{t \geq 0}$ and $(Y(t))_{t \geq 0}$, respectively.

As described, the model is in essence a pair of interacting branching processes. Markov branching processes with interaction have been much studied; see, for instance, [12] and the references therein. The main purpose of this paper is to study the coexistence of the two, competing populations $X$ and $Y$. Similar types of questions have been studied in many contexts.

Received 14 November 2012; revision received 25 January 2013.

* Postal address: Department of Mathematics, Uppsala University, Box 480, Uppsala, 751 06, Sweden.

** Email address: erik.broman@math.uu.se 
One recent example is the so-called two-type Richardson model. This can be informally described as follows. Consider the graph $\mathbb{Z}^{d}$, and let the two infections (red and blue) start with only one individual each. A site is infected by the red (blue) process at a rate equal to the infection parameter $\lambda_{\mathrm{r}}\left(\lambda_{\mathrm{b}}\right)$ times the number of neighbours infected by the red (blue) process. Furthermore, if a site becomes infected by the red infection, it stays red forever, and similarly if it is infected by the blue infection. The main question is if they can coexist, i.e. if there will be two unbounded components of red and blue sites; see, for instance, [4], [6], and [11].

In [3], much focus was on the study of the extinction probability $\eta$ of the infected process $(Y(t))_{t \geq 0}$. There $\eta$ was taken as an indicator of the 'evolutionary fitness' of the virus. The main result was that, for fixed $\left(p_{k}\right)_{k \geq 0}$ and $\left(\gamma_{k}\right)_{k \geq 0}$ satisfying $\gamma_{0}=0$, the extinction probability $\eta$ is maximized when $\lambda=0$. In fact, it was shown that $\eta$ is increasing in $\lambda$. The main result of this paper concerns the coexistence probability $\zeta$.

Definition 1. We call

$$
\zeta=\mathbb{P}(X(t) Y(t) \geq 1 \text { for all } t \geq 0)
$$

the coexistence probability of $X(t), Y(t)$.

Of course, $\zeta$ depends on the parameters used to define the process, but we suppress this dependence in the notation. Introducing the stopping time $T_{\mathrm{u}}=\inf \{t \geq 0: X(t) Y(t)=0\}$, we have $\zeta=\mathbb{P}\left(T_{\mathrm{u}}=\infty\right)$. The relevance of coexistence in the study of $\eta$ will be discussed in Section 6.

The proof of our main result uses two auxiliary branching processes $\hat{X}(t)$ and $\hat{Y}(t)$ defined and discussed in detail in Section 3.1. Informally, $\hat{X}(t)$ is a process distributed as $X(t)$ without the influence of $Y(t)$, i.e. $Y(0)=0$. Furthermore, $\hat{Y}(t)$ is a process distributed as $Y(t)$ with an infinite supply of healthy cells, i.e. $X(0)=\infty$. Our main result is formulated in terms of the so-called Malthusian parameters for these processes, denoted by $\alpha$ and $\beta$ for $\hat{X}(t)$ and $\hat{Y}(t)$, respectively (see Section 3.1). It turns out that $\alpha=\sum_{k=0}^{\infty} k p_{k}-1$ and $\beta=\alpha+p_{0} \sum_{k=0}^{\infty} k \gamma_{k}+\lambda\left(\sum_{k=0}^{\infty} k \gamma_{k}-1\right)$.

Theorem 1. For arbitrary initial conditions $X(0), Y(0) \geq 1$ and offspring distributions $\left(p_{k}\right)_{k \geq 0}$ with finite second moment, the coexistence probability is positive if and only if $\alpha>\beta>0$.

Remark 1. Note that coexistence is only possible if $\gamma_{0}>0$, because otherwise $\beta>\alpha$. This follows from the expressions for $\alpha$ and $\beta$ and Theorem 1: if $\gamma_{0}=0$ then $Y$ cannot die out as long as $X$ survives. Hence, there is then almost surely a time $t>0$ such that $X(t)=0$ and $Y(t) \neq 0$. (This result was announced in [3] as part of Proposition 3.2.)

Theorem 1 establishes, under a second moment condition, for which values of $\alpha$ and $\beta$ we can have coexistence. Our next result strengthens the second part of Theorem 1. Recall that $T_{\mathrm{u}}=\inf \{t>0: X(t) Y(t)=0\}$.

Theorem 2. For offspring distributions $\left(p_{k}\right)_{k \geq 0}$ with finite second moment, and for any choice of $\alpha<\beta$, we have $\mathbb{E}\left[T_{\mathrm{u}}\right]<\infty$.

We have not been able to establish in general if $T_{\mathrm{u}}$ has finite or infinite expectation when $\alpha=\beta$. (However, see Remark 2 below for a special case.)

On the way to proving that coexistence is indeed possible (when $\alpha>\beta$ ) we use general facts about order statistics and trimmed sums; see Lemma 4 below. The second part of that lemma is an interesting application of Harris' inequality [8] to bound the variance of a trimmed sum, which we have not found in the literature. 
In the two-type Richardson model mentioned above, coexistence is conjectured to hold if and only if $\lambda_{\mathrm{r}}=\lambda_{\mathrm{b}}$. The 'if' condition has been established, see [4], [6], and [11], while Häggström and Pemantle [7] made progress on the 'only if' condition. In fact, the model studied here is closer to the following variant of the two-type Richardson model. If a site is infected by the blue process, it changes colour if the red process attempts to infect it, while if a site is infected by the red process it stays so forever. That is, a red site is immune to the blue process, while a blue site is not immune to the red infection. Analogy with the model studied in this paper suggests that there can then be coexistence if $\lambda_{\mathrm{b}}>\lambda_{\mathrm{r}}$, but not if $\lambda_{\mathrm{b}}<\lambda_{\mathrm{r}}$.

We end this section with an outline of the rest of the paper. In Section 2 we give a precise definition of the model. In Section 3 we state and prove the preliminary results needed in the proofs of our main results. In Sections 4 and 5 we prove Theorems 1 and 2, respectively. Finally, in Section 6 we discuss some applications of these results.

\section{Definition of the model}

We briefly recall the stochastic model introduced in [3]. Let $\left(p_{k}\right)_{k \geq 0}$ and $\left(\gamma_{k}\right)_{k \geq 0}$ be probability distributions on the nonnegative integers, and let $\lambda \geq 0$. We exclude the (degenerate) case when $p_{1}=1$; in fact, the reader may for convenience assume that $p_{1}=0$, since this only amounts to a time change.

The continuous-time Markov chain $(X(t), Y(t))_{t \geq 0}$, taking values in $\mathbb{Z}_{+}^{2}$, was informally described in Section 1. To recapitulate the main points, each healthy cell is replaced by $k \geq 0$ new healthy cells at rate $p_{k}$. Being replaced by $k=0$ new cells corresponds to dying. Each infected cell is replaced by $k \geq 1$ new infected cells at rate $p_{k}$. When an infected cell dies, which occurs at rate $p_{0}+\lambda$, a random number of healthy cells are converted into infected cells. This is called a lysis (see transition rate (iii) in Table 1). If $t$ is the time of such an event, we draw a random variable $\Gamma_{t}$ from the distribution $\left(\gamma_{k}\right)_{k \geq 0}$ independently of other events. If $\Gamma_{t} \leq X(t)$, we simply declare $\Gamma_{t}$ of the previously healthy cells to be infected, while if $\Gamma_{t}>X(t)$, we declare all previously healthy cells to be infected. To define this process formally, we list the different possible jumps in Table 1, where we use the notation $x \wedge k=\min \{x, k\}$.

Note that there may be several transitions in Table 1 leading to the same state. In such cases the correct interpretation is to add the corresponding rates. An example of this is the transition $(0, y) \rightarrow(0, y-1)$ that occurs at rate $y\left(p_{0}+\lambda\right)$, which is the sum over all $k \geq 0$ in (iii). To avoid trivial cases, we assume throughout that $X(0), Y(0) \geq 1$. Biologically, it might be most relevant to consider the case when $p_{k}=0$ for $k \geq 3$, but all our results are valid in greater generality, so we make no such restriction.

We now state some immediate properties of the model. If it were the case that $Y(t)=0$, then healthy cells would evolve as a Markov branching process, with intensity 1 and offspring distribution $\left(p_{k}\right)_{k \geq 0}$. Similarly, if $X(t)=0$ for some $t$ then $(Y(t+s))_{s \geq 0}$ would behave like a Markov branching process with the higher intensity $1+\lambda$ and an offspring distribution derived

TABLE 1: Transition rates for the process $(X(t), Y(t))_{t \geq 0}$. Rates are given for transitions from a state $(x, y)$ and are valid for all $x, y \geq 0$.

\begin{tabular}{cccc}
\hline & Transition from $(x, y)$ to & Rate & Valid for \\
\hline (i) & $(x-1+k, y)$ & $x p_{k}$ & $k \geq 0$ \\
(ii) & $(x, y-1+k)$ & $y p_{k}$ & $k \geq 1$ \\
(iii) & $(x-(x \wedge k), y-1+(x \wedge k))$ & $y\left(p_{0}+\lambda\right) \gamma_{k}$ & $k \geq 0$ \\
\hline
\end{tabular}


from $\left(p_{k}\right)_{k \geq 0}$ by placing more mass on $k=0$. When both $X(t), Y(t)>0$, as transition rate (iii) tells us, healthy cells may turn into infected cells. This scenario hence 'helps' the process $(Y(t))_{t \geq 0}$ and 'hurts' the process $(X(t))_{t \geq 0}$.

\section{Preliminary results}

In this section we establish several lemmas which will be used in the proofs of Theorems 1 and 2. Although their motivation may not be obvious on a first reading, we find it convenient to collect all such preliminary results here so as not to interrupt the flow of the main proofs later.

A note on notation: we will sometimes write a sum of the form $\sum_{k=1}^{a} x_{k}$, where $a$ is a noninteger. The correct interpretation is that the sum goes to the integer part $\lfloor a\rfloor$, but we prefer to omit the $\lfloor\cdot\rfloor$ to keep the notation more readable. A similar comment applies also in other places throughout the paper.

\subsection{Auxiliary random variables}

It will at several points be useful to compare $X$ and $Y$ to two 'larger' processes $\hat{X}$ and $\hat{Y}$. Here $\hat{X}$ may be thought of as the healthy process in the absence of infection, and $\hat{Y}$ as the infected process in an infinite 'sea' of healthy cells.

To be precise, we let $\hat{X}$ and $\hat{Y}$ be two branching processes with life length intensities 1 and $1+\lambda$, and offspring distributions $\left(p_{k}\right)_{k \geq 0}$ and $\left(q_{k}\right)_{k \geq 0}$, respectively, where $\left(q_{k}\right)_{k \geq 0}$ is given by

$$
q_{0}=\frac{\gamma_{0}\left(p_{0}+\lambda\right)}{1+\lambda} \quad \text { and } \quad q_{k}=\frac{p_{k}+\gamma_{k}\left(p_{0}+\lambda\right)}{1+\lambda} \quad \text { for } k \geq 1 .
$$

In Table 2 we give a list of the rates used for the coupling of $(\hat{X}, \hat{Y})$ to $(X, Y)$. However, before that, we give an intuitive explanation.

We start with equal size processes, $\hat{X}(0)=X(0)$ and $\hat{Y}(0)=Y(0)$. Each individual in $X(0)$ is paired with a unique 'friend' in $\hat{X}(0)$, and each individual in $Y(0)$ is paired with a unique friend in $\hat{Y}(0)$. Whenever a cell in $X$ either multiplies or dies a natural death (transition (i) in Table 1) then its friend in $\hat{X}$ undergoes the exact same transition, and the offspring are paired in the natural way. Similarly, whenever a cell in $Y$ multiplies (transition (ii) in Table 1) then its friend in $\hat{Y}$ undergoes the exact same transition, and again the offspring are paired in the natural way. When a cell $Y$ has a lysis (transition (iii) in Table 1), sample a random variable $\Gamma$ with distribution $\left(\gamma_{k}\right)_{k \geq 0}$. Infect $\Gamma \wedge X$ cells from $X$, but let the friends in $\hat{X}$ of the newly infected cells in $X$ remain unchanged (but lose their friends, existing as singletons). Proceed by letting the friend in $\hat{Y}$ of the cell in $Y$ which underwent lysis be replaced by $\Gamma$ new cells. Finally, pair the newly infected cells, now belonging to $Y$, with the new cells of $\hat{Y}$. Note that, if $\Gamma>X$,

TABLE 2: Transition rates in the coupled chain $(X, \hat{X}, Y, \hat{Y})$. Rates are given for transitions from a state $(x, \hat{x}, y, \hat{y})$ and are valid for all $x, \hat{x}, y, \hat{y} \geq 0$. Note that the ordering $x \leq \hat{x}, y \leq \hat{y}$ is preserved.

\begin{tabular}{cccc}
\hline & Transition from $(x, \hat{x}, y, \hat{y})$ to state & Rate & Valid for \\
\hline (i) & $(x-1+k, \hat{x}-1+k, y, \hat{y})$ & $x p_{k}$ & $k \geq 0$ \\
(ii) & $(x, \hat{x}-1+k, y, \hat{y})$ & $(\hat{x}-x) p_{k}$ & $k \geq 0$ \\
(iii) & $(x, \hat{x}, y-1+k, \hat{y}-1+k)$ & $y p_{k}$ & $k \geq 1$ \\
(iv) & $(x, \hat{x}, y, \hat{y}-1+k)$ & $(\hat{y}-y) p_{k}$ & $k \geq 1$ \\
(v) & $(x-(x \wedge k), \hat{x}, y-1+(x \wedge k), \hat{y}-1+k)$ & $y\left(p_{0}+\lambda\right) \gamma_{k}$ & $k \geq 0$ \\
(vi) & $(x, \hat{x}, y, \hat{y}-1+k)$ & $(\hat{y}-y)\left(p_{0}+\lambda\right) \gamma_{k}$ & $k \geq 0$ \\
\hline
\end{tabular}


this will result in some of the cells in $\hat{Y}$ being unpaired. Thus, every element of $X$ always has a friend in $\hat{X}$, and every element of $Y$ always has a friend in $\hat{Y}$; but some cells in $\hat{X}$ and $\hat{Y}$ might be unpaired. We let unpaired cells give rise to independent Markov branching processes with the correct intensities and offspring distributions. The rates of the coupled process $(X, \hat{X}, Y, \hat{Y})$ are summarized in Table 2. As before, the correct interpretation is to add the rates of transitions leading to the same state. We note that our coupling satisfies the following statements.

1. $X(t) \leq \hat{X}(t)$ and $Y(t) \leq \hat{Y}(t)$ for all $t \geq 0$;

2. if $X(t) \neq 0$ then $\hat{Y}(t)=Y(t)$.

For a probability vector $\pi=\left(\pi_{k}: k \geq 0\right)$, we write $\bar{\pi}$ for the mean $\sum_{k \geq 0} k \pi_{k}$. Let $\alpha$ and $\beta$ be the Malthusian parameters of $\hat{X}$ and $\hat{Y}$, respectively, given by

$$
\alpha=\bar{p}-1, \quad \beta=(\bar{q}-1)(1+\lambda) .
$$

It is well known [2, Theorem III.7.1] that $\hat{X}(t) / \mathrm{e}^{\alpha t}$ and $\hat{Y}(t) / \mathrm{e}^{\beta t}$ are martingales which converge almost surely to some nonnegative random variables. We have $\mathbb{P}(A \cup B)=1$, where

$$
A=\{\hat{X}(t)=0 \text { for some } t \geq 0\} \quad \text { and } \quad B=\left\{\liminf _{t \rightarrow \infty} \frac{\log (\hat{X}(t))}{t}>0\right\} .
$$

Moreover, $\mathbb{P}(A)=1$ if and only if $\alpha \leq 0$. On the event $B$, the $\operatorname{limit}_{t \rightarrow \infty} \log (\hat{X}(t)) / t$ exists and equals $\alpha$. The corresponding statements hold for $\hat{Y}(t)$ with $\alpha$ replaced by $\beta$. Note for future reference that

$$
\begin{aligned}
\beta & =(\bar{q}-1)(1+\lambda) \\
& =\left(\frac{\bar{p}+\bar{\gamma}\left(p_{0}+\lambda\right)}{1+\lambda}-1\right)(1+\lambda) \\
& =\bar{p}-1+p_{0} \bar{\gamma}+\lambda(\bar{\gamma}-1) \\
& =\alpha+p_{0} \bar{\gamma}+\lambda(\bar{\gamma}-1) .
\end{aligned}
$$

Next, let $U, V, W$, and $\Phi$ denote random variables with the following distributions. Firstly, $U$ and $V$ have the distributions of (the sizes of) $\hat{X}(1)$ and $\hat{Y}(1)$, respectively, when $\hat{X}(0)=1$ and $\hat{Y}(0)=1$. Secondly, $W$ has the distribution of $\tilde{X}(1)$, where $\tilde{X}$ is a branching process, started at 1 , with life length intensity 1 and offspring distribution $\pi$ given by $\pi_{0}=0, \pi_{1}=p_{0}+p_{1}$, and $\pi_{k}=p_{k}$ for $k \geq 2$. Thus, $\tilde{X}$ is essentially $\hat{X}$ with deaths suppressed. Finally, to define $\Phi$, let $t_{1}, t_{2}, \ldots$ denote the times of transitions of the form (v) or (vi) in Table 2 starting with $\hat{Y}(0)=1$, and let $\Gamma_{t_{j}}$ be independent copies of $\Gamma$ as in Section 2. Let $L=\inf \left\{j \geq 1: t_{j}>1\right\}-1$ and $\Phi=\sum_{j=1}^{L} \Gamma_{t_{j}}$. Thus, $\Phi$ is, intuitively, the number of infection attempts during a unit time interval starting with one infected cell.

Lemma 1. Let $r \geq 1$, and let $D$ denote a random variable with distribution $\left(p_{k}\right)_{k \geq 0}$. Then

1. $\mathbb{E}\left(U^{r}\right)<\infty$ if $\mathbb{E}\left(D^{r}\right)<\infty$;

2. $\mathbb{E}\left(V^{r}\right)<\infty$ if $\mathbb{E}\left(D^{r}\right)<\infty$ and $\mathbb{E}\left(\Gamma^{r}\right)<\infty$;

3. $\mathbb{E}\left(W^{r}\right)<\infty$ if $\mathbb{E}\left(D^{r}\right)<\infty$;

4. $\mathbb{E}(\Phi)<\infty$ if $\mathbb{E}(\Gamma)<\infty$ and $\mathbb{E}(D)<\infty$. 
Proof. From [2, Corollary III.6.1], we know that a branching process with offspring distribution $\pi$ has finite $r$ th moment at time $t>0$ if $\pi$ has its $r$ th moment. Hence, parts 1 and 3 are immediate. Part 2 follows from (1), which implies that $\left(q_{k}\right)_{k \geq 0}$ has its $r$ th moment if $\left(p_{k}\right)_{k \geq 0}$ and $\left(\gamma_{k}\right)_{k \geq 0}$ do. For the final part, by Wald's equation, $\mathbb{E}(\Phi)=\mathbb{E}\left(\sum_{j=1}^{L} \Gamma_{t_{j}}\right)=\mathbb{E}(\Gamma) \mathbb{E}(L)$. An easy (stochastic) upper bound on $L$ is given by $\tilde{Y}(1)$, where $\tilde{Y}$ is a branching process with intensity $1+\lambda$ and offspring distribution $\left(\tilde{q}_{k}\right)_{k \geq 0}$, where $\tilde{q}_{0}=\tilde{q}_{1}=0, \tilde{q}_{2}=q_{2}+q_{1}+q_{0}$, and $\tilde{q}_{k}=q_{k}$ for $k \geq 3$. Thus, $\mathbb{E}(L)$ is finite if $\mathbb{E}(\Gamma)$ and $\mathbb{E}(D)$ are finite, as in part 2. This completes the proof.

We will in what follows always assume that $\left(p_{k}\right)_{k \geq 0}$ has finite second moment, since this is part of the assumptions in Theorems 1 and 2. By Lemma 1, this implies that $\mathbb{E}\left(U^{2}\right)<\infty$, $\mathbb{E}\left(V^{2}\right)<\infty, \mathbb{E}\left(W^{2}\right)<\infty$, and $\mathbb{E}(\Phi)<\infty$. This will allow us to apply Chebyshev's bound, which we will use in the following form. Let $Z_{j}, j \geq 1$, be independent, all with the same nonnegative mean $\mathbb{E}(Z) \geq 0$ and finite variance $\operatorname{var}(Z)<\infty$ as some random variable $Z$. Let $N \geq 1$ be any integer, and let $\delta>0$. Then

$$
\begin{aligned}
\mathbb{P}\left(\sum_{j=1}^{N} Z_{j}>(1+\delta) N \mathbb{E}(Z)\right) & \leq \mathbb{P}\left(\left[\sum_{j=1}^{N} Z_{j}-\mathbb{E}\left(Z_{j}\right)\right]^{2}>N^{2} \delta^{2} \mathbb{E}(Z)^{2}\right) \\
& \leq \frac{N \operatorname{var}(Z)}{N^{2} \delta^{2} \mathbb{E}(Z)^{2}} \\
& =\frac{1}{N} \frac{\operatorname{var}(Z)}{\delta^{2} \mathbb{E}(Z)^{2}} .
\end{aligned}
$$

Similarly,

$$
\mathbb{P}\left(\sum_{j=1}^{N} Z_{j}<(1-\delta) N \mathbb{E}(Z)\right) \leq \frac{1}{N} \frac{\operatorname{var}(Z)}{\delta^{2} \mathbb{E}(Z)^{2}}
$$

\subsection{Estimates}

The following lemma says that $Y$ cannot be much larger than $X$ for very long without making $X$ extinct. This lemma will be the main step in the proof of the $\beta>\alpha$ case in Theorem 1 , which is the case in which the process $\hat{Y}$ grows much faster than $X$. In the statement of the lemma, we let $W$ be as in Lemma 1 , and let $\xi$ be a Bernoulli variable with success probability $1-\mathrm{e}^{-\left(1-\gamma_{0}\right)\left(p_{0}+\lambda\right)}$ (this being the probability of a lysis leading to at least one new infection occurring in a time interval of length 1). Fix $c>0$, and let $\delta(t)>0$ be any function such that

$$
n \delta(n)>\frac{1}{2} \log \left(2 \frac{\mathbb{E}(W)}{\mathbb{E}(\xi)}\right)
$$

for all sufficiently large $n$. We write

$$
A_{n}=\left\{\text { for all } t \in[n, n+1], 0<X(t) \leq \mathrm{e}^{(c-\delta(t)) t}<\mathrm{e}^{(c+\delta(t)) t} \leq Y(t)\right\} .
$$

Lemma 2. There exists a constant $C>0$ such that, for $n$ large enough that (6) holds,

$$
\mathbb{P}\left(A_{n}\right) \leq C \mathrm{e}^{-(c-\delta(n)) n} .
$$

In particular, we can take $C=9\left(\operatorname{var}(W) / \mathbb{E}(W)^{2}+\operatorname{var}(\xi) / \mathbb{E}(\xi)^{2}\right)$. It follows that $\mathbb{P}\left(A_{n}\right.$ infinitely often $)=0$.

Before turning to the proof, we remark that we only actually use this lemma with $\delta$ constant. We prove this slightly more general result since very little extra work is required, and we hope that it will be useful for future work. 
Proof of Lemma 2. The result is trivial if $\delta(n) \geq c$, so we assume that $\delta(n)<c$; we also assume throughout the proof that $n$ is large enough that (6) holds. Suppose that $A_{n}$ occurs. Let $\Phi_{n}$ denote the number of infection attempts during the time interval $[n, n+1]$, that is, the sum of an independent sample of $\Gamma$ for each lysis of $(Y(t): t \in[n, n+1])$. Let $\xi^{(n)}$ be obtained from $(Y(t): t \in[n, n+1])$ as follows. Start by numbering the elements of $Y(n)$ (arbitrarily); then observe those elements numbered at most $\mathrm{e}^{(c+\delta(n)) n}$ until they undergo a branching event; let $\xi_{j}$ be the indicator of the event that cell $j$ has a branching event which results in a lysis for which the associated $\Gamma$-value is at least $1\left(\xi_{j}=0\right.$ if there is no branching event before time $n+1)$; finally, let $\xi^{(n)}$ be the sum of the $\xi_{j}$. Then $\xi^{(n)}$ has the following properties:

1. $\xi^{(n)} \leq \Phi_{n}$;

2. $\xi^{(n)}$ is a sum of $\mathrm{e}^{(c+\delta(n)) n}$ independent Bernoulli variables, each with success probability $1-\mathrm{e}^{-p_{0}\left(1-\gamma_{0}\right)(1+\lambda)} ;$ and

3. $\xi^{(n)}$ is independent of $(X(t): t \in[n, n+1])$.

Next, let $W^{(n)}$ denote the total number of healthy cells that ever exist in the time interval $[n, n+1]$. Of course, if $W^{(n)} \leq \Phi_{n}$ then $A_{n}$ cannot occur since this would imply that $X(n+1)=0$. We cannot immediately conclude from $X(t) \leq \mathrm{e}^{(c-\delta(t)) t}$ for every $t \in[n, n+1]$ that $W^{(n)}$ is bounded by $\mathrm{e}^{(c-\delta(n+1))(n+1)}$. However, $W^{(n)}$ must be stochastically bounded by the sum of $\mathrm{e}^{(c-\delta(n)) n}$ independent copies $W_{j}$ of the random variable $W$ in Lemma 1. (Recall that $W$ is, intuitively, $\hat{X}(1)$ when deaths are suppressed.) Also, $W^{(n)}$ is independent of $\xi^{(n)}$. Thus, writing $a_{n}=\mathrm{e}^{(c-\delta(n)) n}$ and $b_{n}=\mathrm{e}^{(c+\delta(n)) n}$, we have

$$
\mathbb{P}\left(A_{n}\right) \leq \mathbb{P}\left(W^{(n)}>\xi^{(n)}\right) \leq \mathbb{P}\left(\sum_{j=1}^{a_{n}} W_{j}>\sum_{j=1}^{b_{n}} \xi_{j}\right)=\mathbb{P}\left(\frac{1}{a_{n}} \sum_{j=1}^{a_{n}} W_{j}>\frac{b_{n}}{a_{n}} \frac{1}{b_{n}} \sum_{j=1}^{b_{n}} \xi_{j}\right) .
$$

Note that $b_{n} / a_{n}=\mathrm{e}^{2 n \delta(n)}>\mathrm{e}^{\log (2 \mathbb{E}(W) / \mathbb{E}(\xi))}=2 \mathbb{E}(W) / \mathbb{E}(\xi)$, by (6). We obtain

$$
\begin{aligned}
\mathbb{P}\left(A_{n}\right) & \leq \mathbb{P}\left(\frac{1}{a_{n}} \sum_{j=1}^{a_{n}} W_{j}>2 \frac{\mathbb{E}(W)}{\mathbb{E}(\xi)} \frac{1}{b_{n}} \sum_{j=1}^{b_{n}} \xi_{j}\right) \\
& \leq \mathbb{P}\left(\frac{1}{a_{n}} \sum_{j=1}^{a_{n}} W_{j}>2 \frac{\mathbb{E}(W)}{\mathbb{E}(\xi)} \frac{2}{3} \mathbb{E}(\xi)\right)+\mathbb{P}\left(\frac{2}{3} \mathbb{E}(\xi)>\frac{1}{b_{n}} \sum_{j=1}^{b_{n}} \xi_{j}\right) \\
& \leq \frac{9 \operatorname{var}(W)}{a_{n} \mathbb{E}(W)^{2}}+\frac{9 \operatorname{var}(\xi)}{b_{n} \mathbb{E}(\xi)^{2}},
\end{aligned}
$$

where we used (4) and (5). This gives (7). That $\mathbb{P}\left(A_{n}\right.$ infinitely often) $=0$ follows from the Borel-Cantelli lemma. This completes the proof.

Recall that if $U(t)$ is a Markov branching process with Malthusian parameter $u$ then $W(t)=$ $U(t) / \mathrm{e}^{u t}$ is a martingale. We make no claim as to the originality of the following lemma, yet have not seen it explicitly formulated.

Lemma 3. Let $U(t)$ be a branching process with Malthusian parameter $u>0$, whose offspring distribution has finite second moment.

1. For any $\Delta>0$, we have that

$$
\mathbb{P}(\text { there exists } t \geq 0: W(t) \geq \Delta) \leq \Delta^{-1} \text {. }
$$


2. For each $\varepsilon>0$, there exists some $\kappa>0$ such that, for all $\tau>0$,

$$
\mathbb{P}\left(\text { there exists } t \geq \tau: 0<W(t)<\mathrm{e}^{-\varepsilon t}\right) \leq \mathrm{e}^{-\kappa \tau} .
$$

Proof. The first part is simply a consequence of Doob's submartingale inequality, which gives, for any $T>0$,

$$
\mathbb{P}(\text { there exists } t \in[0, T]: W(t) \geq \Delta)=\mathbb{P}\left(\sup _{0 \leq t \leq T} W(t) \geq \Delta\right) \leq \frac{\mathbb{E}(W(T))}{\Delta}=\Delta^{-1} .
$$

Letting $T \rightarrow \infty$ concludes the proof of this case.

For the second part, we proceed by discretizing. Let $\mu=\mathbb{E}(U(1))=\mathrm{e}^{u}$, and let $W_{n}=$ $U(n) / \mu^{n}$ for every $n \in \mathbb{N}$. It is no loss of generality to assume that $\varepsilon<u / 2$. The limit $W:=$ $\lim _{n} W_{n}$ exists almost surely since $\left(W_{n}\right)_{n \geq 1}$ is a nonnegative martingale. A straightforward and standard calculation (see, for instance, [9, p. 13]) shows that, for any $r>n$,

$$
\mathbb{E}\left(\mu^{n}\left(W_{r}-W_{n}\right)^{2}\right)=\sigma^{2}\left(\mu^{-1}+\mu^{-2}+\cdots+\mu^{-r}\right),
$$

where $\sigma^{2}=\operatorname{var}(U(1))$. Therefore, by Fatou's lemma,

$$
\mathbb{E}\left(\left(W-W_{n}\right)^{2}\right) \leq \liminf _{r \rightarrow \infty} \mathbb{E}\left(\left(W_{r}-W_{n}\right)^{2}\right)=\frac{\sigma^{2}}{\mu-1} \mu^{-n}
$$

for all $n$. Hence, by Markov's inequality,

$$
\mathbb{P}\left(\left|W-W_{n}\right|>\mathrm{e}^{-\varepsilon n}\right) \leq \frac{\mathbb{E}\left(\left(W-W_{n}\right)^{2}\right)}{\mathrm{e}^{-2 \varepsilon n}} \leq \frac{\sigma^{2}}{\mu-1} \mathrm{e}^{-(u-2 \varepsilon) n} .
$$

It is well known (see, for instance, [9, Theorem 8.3]) that there exists a constant $c_{3}>0$ such that, for any interval $I \subset(0, \infty)$, we have $\mathbb{P}(W \in I) \leq c_{3}|I|$. Furthermore, it is also well known $\left[9\right.$, Theorem 8.4] that there exists a constant $c_{4}>0$ such that $\mathbb{P}\left(W=0, W_{n} \neq 0\right) \leq \mathrm{e}^{-c_{4} n}$. Therefore (adjusting $c_{3}$ as necessary),

$$
\begin{aligned}
\mathbb{P}\left(0<W_{n}<\mathrm{e}^{-\varepsilon n}\right) & \leq \mathbb{P}\left(W=0, W_{n}>0\right)+\mathbb{P}\left(0<W<2 \mathrm{e}^{-\varepsilon n}\right)+\mathbb{P}\left(\left|W-W_{n}\right|>\mathrm{e}^{-\varepsilon n}\right) \\
& \leq c_{3}\left(\mathrm{e}^{-c_{4} n}+\mathrm{e}^{-\varepsilon n}+\mathrm{e}^{-(u-2 \varepsilon) n}\right) .
\end{aligned}
$$

Clearly,

$$
\begin{aligned}
\mathbb{P}\left(\text { there exists } s \geq t: 0<W(s)<\mathrm{e}^{-\varepsilon s}\right) \\
\leq \mathbb{P}\left(\text { there exists } n \geq t: 0<W_{n}<\mathrm{e}^{-\varepsilon n / 2}\right) \\
\quad+\mathbb{P}\left(\text { there exists } s \geq t: 0<W(s)<\mathrm{e}^{-\varepsilon s}, \text { for all } n \geq t, W_{n}=0 \text { or } W_{n} \geq \mathrm{e}^{-\varepsilon n / 2}\right) .
\end{aligned}
$$

We have bounded the first probability on the right-hand side of (8). The second probability is bounded above by

$$
\begin{aligned}
& \mathbb{P}\left(\bigcup_{n \geq t}\left\{\text { there exists } s \in[n, n+1]: W(s)<\mathrm{e}^{-\varepsilon n}, W_{n} \geq \mathrm{e}^{-\varepsilon n / 2}\right\}\right) \\
& \quad \leq \sum_{n \geq t} \mathbb{P}\left(\text { there exists } s \in[n, n+1]: W(s)<\mathrm{e}^{-\varepsilon n} \mid W_{n} \geq \mathrm{e}^{-\varepsilon n / 2}\right) \mathbb{P}\left(W_{n} \geq \mathrm{e}^{-\varepsilon n / 2}\right) \\
& \quad \leq \sum_{n \geq t} \mathbb{P}\left(\text { there exists } s \in[n, n+1]: U(s)<\mathrm{e}^{u(n+1)-\varepsilon n} \mid U(n) \geq \mathrm{e}^{u n-\varepsilon n / 2}\right) .
\end{aligned}
$$

It therefore suffices to show that each of the summands is exponentially small in $n$ for large enough $n$. 
To establish this, we take the following point of view. Let $M=U(n)$, and label the particles present at time $n$ by $1,2, \ldots, M$. If particle $j$ has a branching event with zero offspring, we say that particle $j$ is destroyed. If it has a branching event with one or more offspring, we consider particle $j$ to be still present, essentially identifying it with one of its offspring particles. With this convention, we let $A_{j}$ denote the event that particle $j$ is ever destroyed during the time interval $[n, n+1]$. Thus, $\mathbb{P}\left(A_{j}\right)<1$ for all $j$, and the events $A_{j}$ are independent. If $U(s) \leq \mathrm{e}^{u(n+1)-\varepsilon n}$ for some $s \in[n, n+1]$ then at least $M-\mathrm{e}^{u(n+1)-\varepsilon n}$ of the $A_{j}$ events must occur. But, since $M \geq \mathrm{e}^{u n-\varepsilon n / 2}$,

$$
\begin{aligned}
\mathbb{P}\left(\sum_{j=1}^{M} \mathbf{1}_{A_{j}} \geq M-\mathrm{e}^{u(n+1)-\varepsilon n}\right) & \leq \mathbb{P}\left(\sum_{j=1}^{M} \mathbf{1}_{A_{j}} \geq M\left(1-\mathrm{e}^{u} \mathrm{e}^{-\varepsilon n / 2}\right)\right) \\
& \leq \mathbb{P}\left(\sum_{j=1}^{M} \mathbf{1}_{A_{j}} \geq M \mathbb{P}\left(A_{j}\right)(1+\delta)\right)
\end{aligned}
$$

for large enough $n$ and some $\delta>0$. The latter probability is by (4) at most $C / M \leq C \mathrm{e}^{-(u-\varepsilon / 2) n}$. This gives the result.

\subsection{A lemma about order statistics}

The following result will be used in the $\alpha>\beta$ case of Theorem 1 , but may also be of independent interest. The first part essentially goes back to [1] (for the $p=2$ case), but we have not found the second part in the literature.

If $\left(X_{j}\right)_{1 \leq j \leq M}$ is a sequence of identically distributed random variables, we let $X_{(1)} \leq X_{(2)} \leq$ $\cdots \leq X_{(M)}$ denote the order statistics of $\left(X_{j}\right)_{1 \leq j \leq M}$.

Lemma 4. Let $\left(X_{j}\right)_{1 \leq j \leq M}$ be as above, and let $A \subseteq B \subseteq\{1, \ldots, M\}$.

1. If $p>1$ and $\left\|X_{1}\right\|_{p}=\mathbb{E}\left(X_{1}^{p}\right)^{1 / p}<\infty$, then

$$
\mathbb{E}\left(\sum_{j \in A} X_{(j)}\right) \leq\left\|X_{1}\right\|_{p} M^{1 / p} m^{1 / q},
$$

where $m=|A|$ and $1 / p+1 / q=1$.

2. If the $X_{i}$ are independent and $\mathbb{E}\left(X_{1}^{2}\right)<\infty$, then

$$
\operatorname{var}\left(\sum_{j \in A} X_{(j)}\right) \leq \operatorname{var}\left(\sum_{j \in B} X_{(j)}\right) .
$$

Proof. The first part is a consequence of Hölder's inequality:

$$
\begin{aligned}
\mathbb{E}\left(\sum_{j \in A} X_{(j)}\right) & =\mathbb{E}\left(\sum_{j=1}^{M} X_{(j)} \mathbf{1}\{j \in A\}\right) \\
& \leq \mathbb{E}\left(\sum_{j=1}^{M}\left|X_{(j)}\right|^{p}\right)^{1 / p} \mathbb{E}\left(\sum_{j=1}^{M} \mathbf{1}\{j \in A\}\right)^{1 / q} \\
& =\mathbb{E}\left(\sum_{j=1}^{M}\left|X_{j}\right|^{p}\right)^{1 / p}|A|^{1 / q} \\
& =\left\|X_{1}\right\|_{p} m^{1 / q} M^{1 / p}
\end{aligned}
$$


For the second part, let $X$ denote the sequence $\left(X_{1}, \ldots, X_{M}\right)$ and let

$$
f(X)=\sum_{j \in A} X_{(j)} \quad \text { and } \quad g(X)=\sum_{j \in B \backslash A} X_{(j)} .
$$

Note that both $f$ and $g$ are increasing functions in the sense that if $x=\left(x_{1}, \ldots, x_{n}\right)$ and $y=\left(y_{1}, \ldots, y_{n}\right)$ satisfy $x_{i} \leq y_{i}$ for every $i=1, \ldots, n$, then $f(x) \leq f(y)$ and $g(x) \leq g(y)$. Thus, $f(X)-\mathbb{E}(f(X))$ and $g(X)-\mathbb{E}(g(X))$ are also increasing functions. It follows from Harris' inequality that

$$
\begin{aligned}
& \mathbb{E}((f(X)-\mathbb{E}(f(X)))(g(X)-\mathbb{E}(g(X)))) \\
& \quad \geq \mathbb{E}(f(X)-\mathbb{E}(f(X))) \mathbb{E}(g(X)-\mathbb{E}(g(X))) \\
& \quad=0
\end{aligned}
$$

that is,

$$
\operatorname{cov}\left(\sum_{j \in A} X_{(j)}, \sum_{j \in B \backslash A} X_{(j)}\right) \geq 0
$$

It follows that

$$
\begin{aligned}
\operatorname{var}\left(\sum_{j \in B} X_{(j)}\right) & =\operatorname{var}\left(\sum_{j \in A} X_{(j)}+\sum_{j \in B \backslash A} X_{(j)}\right) \\
& =\operatorname{var}\left(\sum_{j \in A} X_{(j)}\right)+\operatorname{var}\left(\sum_{j \in B \backslash A} X_{(j)}\right)+2 \operatorname{cov}\left(\sum_{j \in A} X_{(j)}, \sum_{j \in B \backslash A} X_{(j)}\right) \\
& \geq \operatorname{var}\left(\sum_{j \in A} X_{(j)}\right) .
\end{aligned}
$$

This completes the proof.

Setting $m=1$ in (9) we deduce that $\mathbb{E}\left(X_{(M)}\right)$ is of order at most $M^{1 / p}$ when the $X_{i}$ have finite $p$ th moments. Results of this type, usually formulated for $p=2$, go back to [1], [5], and [10]. Note that (9) is in some sense sharpest when $A=\{M-m+1, \ldots, M\}$ because then the sum consists of the $m$ largest terms; this is the case we will be using. Also, note that if $B=\{1, \ldots, M\}$ then (10) gives

$$
\operatorname{var}\left(\sum_{j \in A} X_{(j)}\right) \leq M \operatorname{var}\left(X_{1}\right) .
$$

\section{Proof of Theorem 1}

Clearly, (by (2)) $\zeta=0$ if either $\alpha \leq 0$ or $\beta \leq 0$, so we assume henceforth that $\alpha, \beta>0$. The proof of Theorem 1 will be divided into the three cases: (i) $\alpha<\beta$, (ii) $\alpha=\beta$, and (iii) $\alpha>\beta$.

(i) $\alpha<\beta$ case. The intuition is that if coexistence were to take place then $Y(t)$ would eventually be much larger than $X(t)$; but then there is a good chance that all healthy cells are infected in, say, time 1 , which would contradict coexistence. To make this intuition exact, let $c=(\alpha+\beta) / 2$ and $\delta=(\beta-\alpha) / 4>0$, and use (2) to see that

$$
\begin{aligned}
\mathbb{P}\left(T_{\mathrm{u}}=\infty\right) & =\mathbb{P}\left(T_{\mathrm{u}}=\infty, \text { there exists } t_{0}: \hat{X}(t) \leq \mathrm{e}^{(c-\delta) t}<\mathrm{e}^{(c+\delta) t} \leq \hat{Y}(t) \text { for all } t \geq t_{0}\right) \\
& \leq \mathbb{P}\left(\text { there exists } t_{0}: 0<X(t) \leq \mathrm{e}^{(c-\delta) t}<\mathrm{e}^{(c+\delta) t} \leq Y(t) \text { for all } t \geq t_{0}\right),
\end{aligned}
$$


since on $\left\{T_{\mathrm{u}}=\infty\right\}$ we have $0<X(t) \leq \hat{X}(t)$ and $Y(t)=\hat{Y}(t)$ for every $t \geq 0$. Trivially, the right-hand side is bounded above by $\mathbb{P}\left(A_{n}\right.$ infinitely often $)$, where

$$
A_{n}:=\left\{\text { for all } t \in[n, n+1], 0<X(t) \leq \mathrm{e}^{(c-\delta) t}<\mathrm{e}^{(c+\delta) t} \leq Y(t)\right\} .
$$

But $\mathbb{P}\left(A_{n}\right.$ infinitely often $)=0$ by Lemma 2 .

(ii) $\alpha=\beta$ case. For the case $\alpha=\beta>0$, the intuition is that there will typically be so many infection events that $X$ effectively (i.e. counting losses due to infections) has a strictly larger rate of deaths than $\hat{X}$, allowing us to essentially reduce this case to the $\alpha<\beta$ case. Note that the process

$$
R(t)=\frac{\hat{Y}(t)}{\hat{X}(t)}=\frac{\hat{Y}(t)}{\mathrm{e}^{\alpha t}} \frac{\mathrm{e}^{\alpha t}}{\hat{X}(t)}
$$

converges almost surely to some random variable $R$, since $\hat{Y}(t) / \mathrm{e}^{\alpha t}$ and $\hat{X}(t) / \mathrm{e}^{\alpha t}$ are nonnegative martingales. The limit $R$ may be infinite, but on the event $\left\{T_{\mathrm{u}}=\infty\right\}$ we have $0<R<\infty$. Furthermore, since 0 is an absorbing state for the process $R(t)$, we have (up to a null event) $\left\{T_{\mathrm{u}}=\infty\right\} \subseteq\left\{\inf _{t \geq 0} R(t)>0\right\}$. It follows that, for each $r>0$, we have

$$
\left\{T_{\mathrm{u}}=\infty\right\} \subseteq\left\{0<\inf _{t \geq 0} R(t)<r\right\} \cup G_{r},
$$

where

$$
G_{r}=\left\{\frac{\hat{Y}(t)}{\hat{X}(t)} \geq r \text { for all } t \geq 0\right\} \cap\{X(t) Y(t)>0 \text { for all } t \geq 0\} .
$$

For each $\delta>0$, we may choose $r>0$ sufficiently small so that $\mathbb{P}\left(0<\inf _{t \geq 0} R(t)<r\right) \leq \delta$ and, thus, $\zeta \leq \delta+\mathbb{P}\left(G_{r}\right)$. We aim to show that $\mathbb{P}\left(G_{r}\right)=0$ for each $r>0$; since $\delta>0$ was arbitrary, this will complete the proof.

Fix $\delta, r>0$ as above. We will couple $X, \hat{X}$, and $Y$ to a new process $X^{\prime}$ which is obtained by taking into account some of the effect of $Y$ on $X$. The process $X^{\prime}(t)$ will be a Markov branching process and will satisfy $X^{\prime}(t) \leq \hat{X}(t)$ for all $t \geq 0$. Moreover, on the event $G_{r}$ we will have $X(t) \leq X^{\prime}(t)$ for all $t \geq 0$. We let $X^{\prime}(0)=X(0)$. The rates governing the quadruple $\left(X, X^{\prime}, \hat{X}, Y\right)$ are given in Table 3 , where we have written

$$
\kappa=\kappa\left(x^{\prime}, y\right)=\left(r \frac{x^{\prime}}{y}\right) \wedge 1 .
$$

We note from Table 3 that the triple $(X, \hat{X}, Y)$ has the correct marginal distribution, i.e. as described in Section 3.1. For example, summing the first two lines of the table gives the rate $\left(x \wedge x^{\prime}+x-x \wedge x^{\prime}\right) p_{k}=x p_{k}$ for the transition $x \rightarrow x+k-1$. Similarly, $\hat{x} \rightarrow \hat{x}+k-1$ at a rate given by the sum of the first four lines, and using the fact that $x+x^{\prime}-x \wedge x^{\prime}=x \vee x^{\prime}$, we obtain the correct rate $\hat{x} p_{k}$.

Now consider the marginal distribution for $X^{\prime}$. First note that, since $\kappa \leq r x^{\prime} / y$, the final rate in Table 3 is nonnegative. Adding the rates for the transitions $x^{\prime} \rightarrow x^{\prime}-1$, we find that this transition occurs at rate

$$
x^{\prime}\left(p_{0}+r\left(p_{0}+\lambda\right)\left(1-\gamma_{0}\right)\right) .
$$

Together with the rates for $x^{\prime} \rightarrow x^{\prime}+k-1$ for $k \geq 1$, this means that $X^{\prime}(t)$ is a Markov branching process with life length intensity

$$
1+r\left(p_{0}+\lambda\right)\left(1-\gamma_{0}\right)
$$


TABLE 3: Transition rates in the coupled chain $\left(X, X^{\prime}, \hat{X}, Y\right)$. Rates are given for transitions from a state $\left(x, x^{\prime}, \hat{x}, y\right)$ and are valid for all $x, x^{\prime}, \hat{x}, y \geq 0$.

\begin{tabular}{ccc}
\hline Transition to state & Rate & Valid for \\
\hline$\left(x+k-1, x^{\prime}+k-1, \hat{x}+k-1, y\right)$ & $\left(x \wedge x^{\prime}\right) p_{k}$ & $k \geq 0$ \\
$\left(x+k-1, x^{\prime}, \hat{x}+k-1, y\right)$ & $\left(x-x \wedge x^{\prime}\right) p_{k}$ & $k \geq 0$ \\
$\left(x, x^{\prime}+k-1, \hat{x}+k-1, y\right)$ & $\left(x^{\prime}-x \wedge x^{\prime}\right) p_{k}$ & $k \geq 0$ \\
$\left(x, x^{\prime}, \hat{x}+k-1, y\right)$ & $\left(\hat{x}-x \vee x^{\prime}\right) p_{k}$ & $k \geq 0$ \\
$\left(x, x^{\prime}, \hat{x}, y+k-1\right)$ & $y p_{k}$ & $k \geq 1$ \\
$\left(x, x^{\prime}, \hat{x}, y-1\right)$ & $y\left(p_{0}+\lambda\right) \gamma_{0}$ & \\
$\left(x-(x \wedge k), x^{\prime}, \hat{x}, y-1+(x \wedge k)\right)$ & $y\left(p_{0}+\lambda\right) \gamma_{k}(1-\kappa)$ & $k \geq 1$ \\
$\left(x-(x \wedge k), x^{\prime}-1, \hat{x}, y-1+(x \wedge k)\right)$ & $y\left(p_{0}+\lambda\right) \gamma_{k} \kappa$ & $k \geq 1$ \\
$\left(x, x^{\prime}-1, \hat{x}, y\right)$ & $\left(r x^{\prime}-\kappa y\right)\left(p_{0}+\lambda\right)\left(1-\gamma_{0}\right)$ & \\
\hline
\end{tabular}

and offspring distribution $p^{\prime}$ given by

$$
p_{0}^{\prime}=\frac{p_{0}+r\left(p_{0}+\lambda\right)\left(1-\gamma_{0}\right)}{1+r\left(p_{0}+\lambda\right)\left(1-\gamma_{0}\right)}, \quad p_{k}^{\prime}=\frac{p_{k}}{1+r\left(p_{0}+\lambda\right)\left(1-\gamma_{0}\right)}, \quad k \geq 1 .
$$

In particular, the Malthusian parameter of $X^{\prime}$ is

$$
\alpha^{\prime}=\left(1+r\left(p_{0}+\lambda\right)\left(1-\gamma_{0}\right)\right)\left(\bar{p}^{\prime}-1\right)=\alpha-r\left(p_{0}+\lambda\right)\left(1-\gamma_{0}\right)<\alpha \text { for } r>0 .
$$

Clearly, $X^{\prime}(t) \leq \hat{X}(t)$ for all $t \geq 0$. On the event $G_{r}$, we also have $\hat{Y}(t)=Y(t)$ for all $t \geq 0$ and $\hat{Y}(t) / \hat{X}(t) \geq r$ for all $t \geq 0$. It follows that, on $G_{r}$, we have

$$
r \leq \frac{\hat{Y}(t)}{\hat{X}(t)}=\frac{Y(t)}{\hat{X}(t)} \leq \frac{Y(t)}{X^{\prime}(t)} \text { for all } t \geq 0,
$$

so that $r X^{\prime}(t) / Y(t) \leq 1$ and, hence, $\kappa\left(X^{\prime}(t), Y(t)\right)=r X^{\prime}(t) / Y(t)$. Thus, the final rate in Table 3 is always 0 on the event $G_{r}$, and, hence, so is the second rate. Therefore, we obtain $G_{r} \subseteq\left\{X(t) \leq X^{\prime}(t)\right.$ for all $\left.t \geq 0\right\}$.

Let $c=\left(\alpha+\alpha^{\prime}\right) / 2$ and $\delta=\left(\alpha-\alpha^{\prime}\right) / 4>0$. Using (2), we therefore deduce that

$\mathbb{P}\left(G_{r}\right) \leq \mathbb{P}\left(\right.$ there exists $t_{0}: 0<X(t) \leq X^{\prime}(t) \leq \mathrm{e}^{(c-\delta) t}<\mathrm{e}^{(c+\delta) t} \leq Y(t)$ for all $\left.t \geq t_{0}\right)$.

By Lemma 2, the probability on the right equals zero. Since $\delta>0$ was arbitrary it follows that $\zeta=0$.

(iii) $\alpha>\beta$ case. The intuition here is that $X(t)$ 'wants' to be of the order $\mathrm{e}^{\alpha t}$ and $Y(t)$ 'wants' to be of the, much smaller, order $\mathrm{e}^{\beta t}$. Typically, therefore, the infection will have very little impact on the healthy population.

To make this intuition rigorous, let

$$
a_{n}=\prod_{k=2}^{n}\left(1-\frac{2}{k^{2}}\right), \quad b_{n}=\prod_{k=2}^{n}\left(1-\frac{1}{k^{2}}\right), \quad c_{n}=\prod_{k=2}^{n}\left(1+\frac{1}{k^{2}}\right) .
$$

Note that $a_{n}$ and $b_{n}$ form decreasing sequences with limits in $(0,1)$ and that $c_{n}$ is an increasing sequence with limit in $(1, \infty)$. Write $B_{n}$ for the event that

$$
X(n) \geq a_{n} \mathrm{e}^{\alpha n} \quad \text { and } \quad b_{n} \mathrm{e}^{\beta n} \leq Y(n) \leq c_{n} \mathrm{e}^{\beta n} .
$$


We will prove that there is some $N$ such that

$$
\mathbb{P}\left(B_{n+1} \mid B_{n}\right) \geq 1-\frac{3}{n^{2}}
$$

for all $n \geq N$. This will, using the Markov property, establish the result, since $\mathbb{P}\left(B_{N}\right)>0$ and

$$
\zeta \geq \mathbb{P}\left(\bigcap_{n \geq N} B_{n}\right)=\mathbb{P}\left(B_{N}\right) \prod_{n \geq N} \mathbb{P}\left(B_{n+1} \mid B_{n}\right)>0 .
$$

We start by observing that (again using the fact that $Y(t)=\hat{Y}(t)$ whenever $X(t)>0$ )

$$
\begin{aligned}
\mathbb{P}\left(B_{n+1}\right. & \left.\mid B_{n}\right) \\
= & \mathbb{P}\left(X(n+1) \geq a_{n+1} \mathrm{e}^{\alpha(n+1)}, b_{n+1} \mathrm{e}^{\beta(n+1)} \leq \hat{Y}(n+1) \leq c_{n+1} \mathrm{e}^{\beta(n+1)} \mid B_{n}\right) \\
\geq & 1-\mathbb{P}\left(X(n+1)<a_{n+1} \mathrm{e}^{\alpha(n+1)} \mid B_{n}\right)-\mathbb{P}\left(\hat{Y}(n+1)<b_{n+1} \mathrm{e}^{\beta(n+1)} \mid B_{n}\right) \\
& -\mathbb{P}\left(\hat{Y}(n+1)>c_{n+1} \mathrm{e}^{\beta(n+1)} \mid B_{n}\right) .
\end{aligned}
$$

We will proceed to show that all three probabilities on the right-hand side are small. To prove that $\mathbb{P}\left(X(n+1)<a_{n+1} \mathrm{e}^{\alpha(n+1)} \mid B_{n}\right)$ is small, let $\Phi_{n}$ denote the number of infection attempts during the time interval $[n, n+1]$, as in the proof of Lemma 2. We will first show that $\Phi_{n}$ will typically be much smaller than $X(n)$, and will deduce from this the required lower bound on $X(n+1)$. For the bound on $\Phi_{n}$, we use Markov's inequality to see that

$$
\mathbb{P}\left(\Phi_{n} \geq c_{n} \mathrm{e}^{\beta n}(n+1)^{2} \mathbb{E}(\Phi) \mid B_{n}\right) \leq \frac{\mathbb{E}\left(\Phi_{n} \mid B_{n}\right)}{c_{n} \mathrm{e}^{\beta n}(n+1)^{2} \mathbb{E}(\Phi)} \leq \frac{1}{(n+1)^{2}},
$$

where $\Phi$ is the random variable of Lemma 1 , and where we used the fact that, given $B_{n}$, the number $\Phi_{n}$ of infection attempts is dominated by the sum of $c_{n} \mathrm{e}^{\beta n}$ independent copies of $\Phi$.

Let $M=M(n)=a_{n} \mathrm{e}^{\alpha n}$ and $m=m(n)=c_{n} \mathrm{e}^{\beta n}(n+1)^{2} \mathbb{E}(\Phi)$ (so $X(n) \geq M$ on $B_{n}$, and $m$ is the quantity in (14)). Let $\left(U_{j}\right)_{1 \leq j \leq M}$ denote independent copies of the random variable $U$ of Lemma 1 . The lower bound on $X(n+1)$ will be obtained by noting that the impact of infection during the time interval $[n, n+1]$ can be no larger than the effect of removing, at time $n$, those $\Phi_{n}$ healthy cells that would otherwise give rise to the largest ancestry at time $n+1$. In particular,

$$
X(n+1) \geq \sum_{j=1}^{X(n)-\Phi_{n}} U_{(j)}
$$

where $U_{(1)} \leq U_{(2)} \leq \cdots \leq U_{(M)}$ denote the order statistics of $U_{1}, \ldots, U_{M}$ as in Section 3.3. For large enough $n$, we have $M \geq m$, and on the event $B_{n} \cap\left\{\Phi_{n} \leq m\right\}$ we have

$$
X(n+1) \geq \sum_{j=1}^{M-m} U_{(j)}
$$

Recall that $\mathbb{E}\left(U_{j}\right)=\mathrm{e}^{\alpha}$. From the first part of Lemma 4, we have

$$
\mathbb{E}\left(\sum_{j=M-m+1}^{M} U_{(j)}\right)=O(\sqrt{m M})=O\left(M \frac{n}{\mathrm{e}^{(\alpha-\beta) n / 2}}\right),
$$

where $O(f(n))$ denotes a function bounded above by $C f(n)$ for some $0<C<\infty$. 
Observe that $a_{n+1} \mathrm{e}^{\alpha(n+1)}=\left(1-2 /(n+1)^{2}\right) M \mathrm{e}^{\alpha}$ and that, for large enough $n$, we have

$$
\begin{aligned}
& \mathbb{P}\left(\sum_{j=1}^{M-m} U_{(j)}<\left(1-\frac{2}{(n+1)^{2}}\right) M \mathrm{e}^{\alpha}\right) \\
& \quad=\mathbb{P}\left(\sum_{j=1}^{M-m} U_{(j)}-\mathbb{E}\left(\sum_{j=1}^{M} U_{j}\right)<-\frac{2 M \mathrm{e}^{\alpha}}{(n+1)^{2}}\right) \\
& \quad=\mathbb{P}\left(\sum_{j=1}^{M-m} U_{(j)}-\mathbb{E}\left(\sum_{j=1}^{M-m} U_{(j)}\right)<-\frac{2 M \mathrm{e}^{\alpha}}{(n+1)^{2}}+\mathbb{E}\left(\sum_{j=M-m+1}^{M} U_{(j)}\right)\right) \\
& \quad \leq \mathbb{P}\left(\sum_{j=1}^{M-m} U_{(j)}-\mathbb{E}\left(\sum_{j=1}^{M-m} U_{(j)}\right)<-\frac{M \mathrm{e}^{\alpha}}{(n+1)^{2}}\right) .
\end{aligned}
$$

By Chebyshev's bound, (4), and the second part of Lemma 4, with $A=\{1, \ldots, M-m\}$ and $B=\{1, \ldots, M\}$,

$$
\begin{aligned}
\mathbb{P}\left(\sum_{j=1}^{M-m} U_{(j)}-\mathbb{E}\left(\sum_{j=1}^{M-m} U_{(j)}\right)<-\frac{M \mathrm{e}^{\alpha}}{(n+1)^{2}}\right) & \leq \frac{(n+1)^{4} \operatorname{var}\left(\sum_{j=1}^{M-m} U_{(j)}\right)}{\mathrm{e}^{2 \alpha} M^{2}} \\
& \leq \frac{(n+1)^{4} \operatorname{var}\left(U_{1}\right)}{\mathrm{e}^{2 \alpha} M} \\
& =O\left(\mathrm{e}^{-\alpha n}\right) .
\end{aligned}
$$

Taking into account also (14), it follows that

$$
\begin{aligned}
\mathbb{P}\left(X(n+1) \geq a_{n+1} \mathrm{e}^{\alpha(n+1)} \mid B_{n}\right) \\
\quad \geq \mathbb{P}\left(X(n+1) \geq a_{n+1} \mathrm{e}^{\alpha(n+1)} \mid \Phi_{n} \leq m, B_{n}\right) \mathbb{P}\left(\Phi_{n} \leq m \mid B_{n}\right) \\
\quad \geq \mathbb{P}\left(\sum_{j=1}^{M-m} U_{(j)} \geq a_{n+1} \mathrm{e}^{\alpha(n+1)}\right)\left(1-\frac{1}{(n+1)^{2}}\right) \\
\quad \geq\left(1-O\left(\mathrm{e}^{-\alpha n}\right)\right)\left(1-\frac{1}{(n+1)^{2}}\right) \\
\quad \geq 1-\frac{2}{(n+1)^{2}}
\end{aligned}
$$

for large enough $n$.

We proceed with the second and third probabilities on the right-hand side of (13). With $V_{j}$ independent and having the distribution of $V$ in Lemma 1, using the fact that $\mathbb{E}(V)=\mathrm{e}^{\beta},(5)$, and the fact that $Y(t)=\hat{Y}(t)$ whenever $X(t)>0$, we have

$$
\begin{aligned}
& \mathbb{P}\left(\hat{Y}(n+1)<b_{n+1} \mathrm{e}^{\beta(n+1)} \mid B_{n}\right) \\
& \quad=\mathbb{P}\left(\hat{Y}(n+1)<b_{n+1} \mathrm{e}^{\beta(n+1)} \mid X(n) \geq a_{n} \mathrm{e}^{\alpha n}, b_{n} \mathrm{e}^{\beta n} \leq \hat{Y}(n) \leq c_{n} \mathrm{e}^{\beta n}\right) \\
& \quad=\mathbb{P}\left(\hat{Y}(n+1)<b_{n+1} \mathrm{e}^{\beta(n+1)} \mid b_{n} \mathrm{e}^{\beta n} \leq \hat{Y}(n) \leq c_{n} \mathrm{e}^{\beta n}\right) \\
& \quad \leq \mathbb{P}\left(\hat{Y}(n+1)<b_{n+1} \mathrm{e}^{\beta(n+1)} \mid \hat{Y}(n)=b_{n} \mathrm{e}^{\beta n}\right)
\end{aligned}
$$




$$
\begin{aligned}
& =\mathbb{P}\left(\sum_{j=1}^{b_{n} \mathrm{e}^{\beta n}} V_{j}<b_{n+1} \mathrm{e}^{\beta(n+1)}\right) \\
& =\mathbb{P}\left(\sum_{j=1}^{b_{n} \mathrm{e}^{\beta n}} V_{j}<\left(1-\frac{1}{(1+n)^{2}}\right) b_{n} \mathrm{e}^{\beta n} \mathbb{E}(V)\right) \\
& \leq \frac{1}{b_{n} \mathrm{e}^{\beta n}} \frac{(1+n)^{4} \operatorname{var}(V)}{\mathbb{E}(V)^{2}} \\
& =O\left(\mathrm{e}^{-\beta n}\right) .
\end{aligned}
$$

Similarly, but using (4) in place of (5),

$$
\begin{aligned}
\mathbb{P}\left(\hat{Y}(n+1)>c_{n+1} \mathrm{e}^{\beta(n+1)} \mid B_{n}\right) & \leq \mathbb{P}\left(\hat{Y}(n+1)>c_{n+1} \mathrm{e}^{\beta(n+1)} \mid \hat{Y}(n)=c_{n} \mathrm{e}^{\beta n}\right) \\
& =\mathbb{P}\left(\sum_{j=1}^{c_{n} \mathrm{e}^{\beta n}} V_{j}>c_{n+1} \mathrm{e}^{\beta(n+1)}\right) \\
& =\mathbb{P}\left(\sum_{j=1}^{c_{n} \mathrm{e}^{\beta n}} V_{j}>\left(1+\frac{1}{(1+n)^{2}}\right) c_{n} \mathrm{e}^{\beta n} \mathbb{E}(V)\right) \\
& \leq \frac{1}{c_{n} \mathrm{e}^{\beta n}} \frac{(1+n)^{4} \operatorname{var}(V)}{\mathbb{E}(V)^{2}} \\
& =O\left(\mathrm{e}^{-\beta n}\right) .
\end{aligned}
$$

We conclude that (12) holds for large enough $n$.

\section{Proof of Theorem 2}

The proof of Theorem 2 will be carried out in two parts.

(i) $\alpha<0$ case. It is well known (see [9, Theorem 11.1]) that the probability that a subcritical branching process survives until time $t>0$ decays exponentially fast in $t$. That is, there exists $c>0$ such that, for every $t>0$,

$$
\mathbb{P}(X(t)>0) \leq \mathrm{e}^{-c t} .
$$

Letting $T_{X}=\inf \{t: X(t)=0\}$, we obtain $\mathbb{E}\left(T_{\mathrm{u}}\right) \leq \mathbb{E}\left(T_{X}\right)<\infty$.

(ii) $0<\alpha<\beta$ case. Similarly to (11), let $c=(\alpha+\beta) / 2$ and $\delta=(\beta-\alpha) / 4$, and note that $c-\delta=\alpha+\delta$ and $c+\delta=\beta-\delta$. We have

$$
\begin{aligned}
\mathbb{P}\left(T_{\mathrm{u}} \geq \tau\right)= & \mathbb{P}\left(T_{\mathrm{u}} \geq \tau, \hat{X}(t) \leq \mathrm{e}^{(c-\delta) t}<\mathrm{e}^{(c+\delta) t} \leq \hat{Y}(t) \text { for all } t \geq \frac{\tau}{2}\right) \\
& +\mathbb{P}\left(T_{\mathrm{u}} \geq \tau,\left\{\hat{X}(t) \leq \mathrm{e}^{(c-\delta) t}<\mathrm{e}^{(c+\delta) t} \leq \hat{Y}(t) \text { for all } t \geq \frac{\tau}{2}\right\}\right) \\
\leq & \mathbb{P}\left(T_{\mathrm{u}} \geq \tau, \hat{X}(t) \leq \mathrm{e}^{(c-\delta) t}<\mathrm{e}^{(c+\delta) t} \leq \hat{Y}(t) \text { for all } t \geq \frac{\tau}{2}\right) \\
& +\mathbb{P}\left(T_{\mathrm{u}} \geq \tau, \text { there exists } t \geq \frac{\tau}{2}: \hat{X}(t) \geq \mathrm{e}^{(\alpha+\delta) t}\right. \\
& \text { or there exists } \left.t \geq \frac{\tau}{2}: 0<\hat{Y}(t)<\mathrm{e}^{(\beta-\delta) t}\right)
\end{aligned}
$$




$$
\begin{gathered}
\leq \mathbb{P}\left(T_{\mathrm{u}} \geq \tau, \hat{X}(t) \leq \mathrm{e}^{(c-\delta) t}<\mathrm{e}^{(c+\delta) t} \leq \hat{Y}(t) \text { for all } t \geq \frac{\tau}{2}\right) \\
+\mathbb{P}\left(\text { there exists } t \geq \frac{\tau}{2}: \hat{X}(t) \geq \mathrm{e}^{(\alpha+\delta) t}\right. \\
\text { or there exists } \left.t \geq \frac{\tau}{2}: 0<\hat{Y}(t)<\mathrm{e}^{(\beta-\delta) t}\right) .
\end{gathered}
$$

For the first part of the right-hand side of (15), we first consider (for simplicity) the $\tau=2 n$ case, for which we obtain

$$
\begin{aligned}
\mathbb{P}\left(T_{\mathrm{u}}\right. & \left.\geq \tau, \hat{X}(t) \leq \mathrm{e}^{(c-\delta) t}<\mathrm{e}^{(c+\delta) t} \leq \hat{Y}(t) \text { for all } t \geq \frac{\tau}{2}\right) \\
& =\mathbb{P}\left(T_{\mathrm{u}} \geq 2 n, \hat{X}(t) \leq \mathrm{e}^{(c-\delta) t}<\mathrm{e}^{(c+\delta) t} \leq \hat{Y}(t) \text { for all } t \geq n\right) \\
& \leq \mathbb{P}\left(0<X(t) \leq \mathrm{e}^{(c-\delta) t}<\mathrm{e}^{(c+\delta) t} \leq Y(t) \text { for all } t \in[n, n+1]\right) \\
& =\mathbb{P}\left(A_{n}\right)
\end{aligned}
$$

where $A_{n}$ is as in Lemma 2. According to that lemma, there exists a $c_{2}>0$ such that, for any $n$, we have $\mathbb{P}\left(A_{n}\right) \leq \mathrm{e}^{-2 c_{2} n}=\mathrm{e}^{-c_{2} \tau}$. It is easy to see that the same holds for all $\tau$ (adjusting $c_{2}$ if necessary).

For the second part of the right-hand side of (15), we use Lemma 3, to conclude that there exists a $c_{1}=c_{1}(\delta)>0$ such that, for any $\tau$,

$$
\begin{aligned}
& \mathbb{P}\left(\text { there exists } t \geq \frac{\tau}{2}: \hat{X}(t) \geq \mathrm{e}^{(\alpha+\delta) t} \cup \text { there exists } t \geq \frac{\tau}{2}: 0<\hat{Y}(t)<\mathrm{e}^{(\beta-\delta) t}\right) \\
& \quad \leq \mathbb{P}\left(\text { there exists } t \geq \frac{\tau}{2}: \hat{X}(t) \geq \mathrm{e}^{\alpha t+\delta \tau / 2}\right)+\mathbb{P}\left(\text { there exists } t \geq \frac{\tau}{2}: 0<\hat{Y}(t)<\mathrm{e}^{(\beta-\delta) t}\right) \\
& \quad \leq \mathrm{e}^{-\delta \tau / 2}+\mathrm{e}^{-c_{1} \tau} .
\end{aligned}
$$

We conclude that there exists $c_{3}>0$ such that $\mathbb{P}\left(T_{\mathrm{u}} \geq t\right) \leq \mathrm{e}^{-c_{3} t}$ for any $t>0$, and so $\mathbb{E}\left(T_{\mathrm{u}}\right)<\infty$.

Remark 2. Clearly, $\mathbb{E}\left(T_{\mathrm{u}}\right)=\infty$ when $\alpha>\beta>0$, since then $T_{\mathrm{u}}$ takes value $\infty$ with positive probability. We have not been able to determine in general whether or not $\mathbb{E}\left(T_{\mathrm{u}}\right)$ is finite in the remaining case $\alpha=\beta$, but in the following special case it is easily seen to be finite. Suppose that $\alpha=\beta=0, \gamma_{0}=1$, and $\lambda=0$. Then $X$ and $Y$ form independent critical branching processes. The extinction times $T_{X}$ and $T_{Y}$ for these respective processes satisfy

$$
\mathbb{P}\left(T_{X}>t\right) \sim \frac{1}{t}, \quad \mathbb{P}\left(T_{Y}>t\right) \sim \frac{1}{t} ;
$$

see [2, p. 159]. Thus, $T_{\mathrm{u}}=\min \left\{T_{X}, T_{Y}\right\}$ satisfies

$$
\mathbb{P}\left(T_{\mathrm{u}}>t\right)=\mathbb{P}\left(T_{X}>t\right) \mathbb{P}\left(T_{Y}>t\right) \sim \frac{1}{t^{2}},
$$

so

$$
\mathbb{E}\left(T_{\mathrm{u}}\right)=\int_{0}^{\infty} \mathbb{P}\left(T_{\mathrm{u}}>t\right) \mathrm{d} t \leq 1+\int_{1}^{\infty} \mathbb{P}\left(T_{\mathrm{u}}>t\right) \mathrm{d} t \sim 1+\int_{1}^{\infty} \frac{\mathrm{d} t}{t^{2}}<\infty
$$




\section{Applications of the main results}

In this section we will briefly discuss some applications of our main theorems. Using our results on coexistence, we are able to comment more on the issue of extinction of $Y$, which was the main focus of [3].

Central to the analysis in the present article were the auxiliary processes $\hat{X}$ and $\hat{Y}$. Recall that $\hat{Y}$ was in essence the process $Y$ in an 'infinite sea of food', i.e. $X(0)=\infty$. However, if instead $X(t)=0$ then $(Y(t+s))_{s \geq 0}$ has no healthy cells to feed on, and, therefore, $(Y(t+s))_{s \geq 0}$ grows at the exponential rate (see also (3))

$$
\beta^{\prime}=\bar{p}-1-\lambda=\alpha-\lambda
$$

The qualitative behaviour of $(X(t), Y(t))_{t \geq 0}$ depends on the values of $\alpha, \beta$, and $\beta^{\prime}$. We discuss the possible different regimes.

Regime 1. If $\alpha \leq 0$ then $(X(t))_{t \geq 0}$ eventually dies out, and since $\beta^{\prime} \leq \alpha$, so does $(Y(t))_{t \geq 0}$. Hence, $\eta=1$.

Regime 2. If $0<\alpha \leq \beta$ then, if $\gamma_{0}>0$, it might be the case that $(Y(t))_{t \geq 0}$ dies out spontaneously. However, if it does not then, according to Theorem $1,(X(t))_{t \geq 0}$ will instead go extinct. If $\beta^{\prime} \leq 0$, we then conclude that $(Y(t))_{t \geq 0}$ also dies out, that is, $\eta=1$. However, if $\beta^{\prime}>0$ then $(Y(t))_{t \geq 0}$ can survive on its own, that is, $\eta<1$.

Regime 3. If $0<\beta<\alpha$, we are in the coexistence regime, in particular, $\eta<1$. As stated in Theorem 1, it might be the case that $X(t) Y(t)>0$ for all $t>0$. However, as in Regime 2, if $\gamma_{0}>0$, it is possible that $(Y(t))_{t \geq 0}$ dies out. Furthermore, if $(X(t))_{t \geq 0}$ dies out then the behaviour of $(Y(t))_{t \geq 0}$ would again be governed by the sign of $\beta^{\prime}$.

Regime 4. If $\beta<0$ then $(Y(t))_{t \geq 0}$ eventually dies out, that is, $\eta=1$.

We can draw qualitative conclusions from the above description, also using (3). For instance, if we fix $\alpha>0$ and $\mathbb{E}(\Gamma) \geq 1$, it follows that $\alpha \leq \beta$ for every $\lambda \geq 0$, and so we are always in Regime 2. As long as $\lambda$ is small enough, so that $\beta^{\prime}>0$, the process $(Y(t))_{t \geq 0}$ can survive. This supports the intuition that small $\lambda$ is good for the long term survival of $(Y(t))_{t \geq 0}$; see [3].

If instead $\alpha>0$ while $\mathbb{E}(\Gamma)<1$, we see that we are in Regime 2 for small values of $\lambda$ and in Regime 3 for large values of $\lambda$. Depending on the exact values of $\alpha$ and $\mathbb{E}(\Gamma)$, we have the following possibilities:

- for small $\lambda$, we have $0<\alpha<\beta$, and $\beta^{\prime}>0$ so that $(Y(t))_{t \geq 0}$ might survive, that is, $\eta<1$;

- for slightly larger $\lambda$, we can have $0<\alpha<\beta$, and $\beta^{\prime} \leq 0$ so that $(Y(t))_{t \geq 0}$ dies out, that is, $\eta=1$;

- for larger $\lambda$, we have $0<\beta<\alpha$ so that $(Y(t))_{t \geq 0}$ might again survive, that is, $\eta<1$;

- for even larger $\lambda$, we have $\beta \leq 0$ so that $(Y(t))_{t \geq 0}$ again dies out, that is, $\eta=1$.

In [3], monotonicity of $\eta$ as a function of $\lambda$ was established when $\gamma_{0}=0$. In contrast, we see here that monotonicity of $\eta$ in $\lambda$ may fail if $\mathbb{E}(\Gamma)<1$ (and it is easy to find specific parameters for this to be the case). Note also the difference between the first case, in which $(Y(t))_{t \geq 0}$ is strong enough to survive on its own, and the third case where $(Y(t))_{t \geq 0}$ needs the process $(X(t))_{t \geq 0}$ to feed on. 


\section{References}

[1] Arnold, B. C. and Groeneveld, R. A. (1979). Bounds on expectations of linear systematic statistics based on dependent samples. Ann. Statist.7, 220-223.

[2] Athreya, K. B. And Ney, P. E. (1972). Branching Processes. Springer, New York.

[3] Björnberg, J. E., Britton, T., Broman, E. I. and Natan, E. (2012). A stochastic model for virus growth in a cell population. Preprint. Available at http://arxiv.org/abs/1112.4694v2.

[4] Garet, O. and Marchand, R. (2005). Coexistence in two-type first-passage percolation models. Ann. Appl. Prob. 15, 298-330.

[5] Gumbel, E. J. (1954). The maxima of the mean largest value and of the range. Ann. Math. Statist. 25, 76-84.

[6] Häggström, O. and Pemantle, R. (1998). First passage percolation and a model for competing spatial growth. J. Appl. Prob. 35, 683-692.

[7] Häggström, O. and Pemantle, R. (2000). Absence of mutual unbounded growth for almost all parameter values in the two-type Richardson model. Stoch. Process. Appl. 90, 207-222.

[8] Harris, T. E. (1960). A lower bound for the critical probability in a certain percolation process. Proc. Camb. Phil. Soc. 56, 13-20.

[9] Harris, T. E. (1963). The Theory of Branching Processes. Springer, Berlin.

[10] Hartley, H. O. and David, H. A. (1954). Universal bounds for mean range and extreme observation. Ann. Math. Statist. 25, 85-99.

[11] Hoffman, C. (2005). Coexistence for Richardson type competing spatial growth models. Ann. Appl. Prob. 15, 739-747.

[12] Kalinkin, A. V. (2002). Markov branching processes with interaction. Russian Math. Surveys 57, $241-304$. 